\title{
High Incidence of Thrombosis in Fabry's Disease
}

\author{
Kouichi Utsumi, Naomasa Yamamoto*, Ryoichi Kase, Tomoyo Takata, Toshika OkumiYa, \\ Hiroshi SaIto**, Tadashi SuzukI***, Eiichiro Uyama**** and Hitoshi SaKuraba
}

\begin{abstract}
Although a high incidence of thrombotic accidents in Fabry's disease has been postulated, few investigations have been performed. To clarify the incidence of thrombosis in Fabry's disease, we undertook a systematic study on thrombosis in patients with Fabry's disease including hemizygous males and heterozygous females. Sixty patients with Fabry's disease (45 hemizygotes and 15 heterozygotes) from 36 Japanese families were subjected to clinical, biochemical and genetic investigations. We found that seven patients with Fabry's disease (4 hemizygous males and 3 heterozygous females) had experienced thrombotic accidents. Six of these thrombotic patients developed brain infarctions, including one man who had the complication of recurrent thrombophlebitis. The remaining woman showed central retinal artery occlusion and thrombophlebitis. We demonstrated a high incidence of thrombosis in Fabry's disease. Thrombotic accidents occurred not only in hemizygous males but also in heterozygous females. The complication of thrombotic accidents should be taken into account in patients with Fabry's disease.
\end{abstract}

(Internal Medicine 36: 327-329, 1997)

Key words: brain infarctions, thrombophlebitis, central retinal occlusion, globotriaosylceramide, $\alpha$-galactosidase activity

\section{Introduction}

Fabry's disease results from lesions in the X-linked gene encoding $\alpha$-galactosidase $(\alpha-G a l)$, which lead to the systemic accumulation of globotriaosylceramide (1). Patients with the classic form of early-onset show diverse clinical manifestations, such as pain and paresthesia in the distal extremities, angiokeratoma, hypohidrosis, corneal opacity, and progressive vasculopathy in the kidneys, heart and central nervous system. Recently, a high frequency of patients with a variant form of the disease, who have residual $\alpha$-Gal activity and late-onset cardiomyopathy without other systemic manifestations have been found in Japan (2). Heterozygous females develop a wide range of clinical manifestations, and some patients have been reported to have severe manifestations $(3,4)$. The incidence of classic Fabry's disease is estimated to be about 1 in 40,000 (1). A high incidence of thrombotic accidents in Fabry's disease has been postulated $(1,5)$. However, a systematic study on thrombosis in cases of Fabry's disease, including both hemizygous males and heterozygous females, has not been undertaken.

\section{Patients and Methods}

We screened 60 patients with Fabry's disease from 36 unrelated Japanese families. The patients included 35 males with the classic form, 10 males with the variant form, and 15 heterozygous females from families with the classic form. Fabry's disease was diagnosed on the basis of the clinical signs and symptoms, family pedigree, and $\alpha$-Gal activity. The $\alpha$-Gal activity in leukocytes from the patients was measured using an artificial substrate, 4-methylumbelliferyl $\alpha$-D-galactoside, as described by Mayes et al (6). Mutations of the $\alpha$-Gal gene in the patients were identified by polymerase chain reaction (PCR) amplification and sequencing as described previously (7). Thrombosis was detected by cranial computed tomography (CT), cranial magnetic resonance imaging (MRI), ophthalmoscopy and venography. The results were expressed as mean \pm SD. Statistical analysis was performed using Student's $t$-test. $P$ values $<0.05$ were considered significant.

From the Department of Clinical Genetics and *the Department of Cardiovascular Research, the Tokyo Metropolitan Institute of Medical Science, Tokyo, **the Department of Nephrology, Tokyo Metropolitan Komagome Hospital, Tokyo, ***the College of Medical Care and Technology, Gunma University, Maebashi and ****the Department of Neurology, Kumamoto University School of Medicine, Kumamoto

Received for publication October 8, 1996; Accepted for publication March 6, 1997

Reprint requests should be addressed to Dr. Hitoshi Sakuraba, the Department of Clinical Genetics, The Tokyo Metropolitan Institute of Medical Science, 3-1822 Honkomagome, Bunkyo-ku, Tokyo 113 


\section{UTSUMI et al}

\section{Results}

We found that 7 patients (12\%) experienced thrombotic accidents at ages 18 to 57, and before age 35 year in 4 cases (Table 1). All 7 patients had classic Fabry's disease (4 hemizygotes and 3 heterozygotes). Six of them had brain infarctions, including a male patient with the complication of recurrent thrombophlebitis. The remaining patient, a woman, showed central retinal artery occlusion and thrombophlebitis in the right lower extremity, which were confirmed by ophthalmoscopy and venography, respectively.

Among the hemizygotes with classic Fabry's disease, there was no difference in leukocyte $\alpha$-Gal activity between the 4 thrombotic patients $(0.5 \pm 0.5 \%$ of the control level $)$ and the 31 non-thrombotic patients $(0.5 \pm 0.4 \%)$. The leukocyte $\alpha$-Gal activity in the 3 heterozygous patients who experienced thrombotic accidents $(17.2 \pm 5.9 \%)$ was significantly $(\mathrm{p}<0.05)$ lower than that in the 12 non-thrombotic heterozygous females (47.1 $\pm 23.1 \%)$. However, the leukocyte $\alpha$-Gal activity in the heterozygous females who experienced thrombotic accidents was significantly higher than that in the 10 hemizygous males with the variant form $(6.7 \pm 4.2 \%)$.

Patient 4 was recently diagnosed, and he has a novel C-to-T transition at cDNA number 1069 in exon 7, resulting in substitution of the predictable termination (TAG) for glutamine
(CAG) at codon 357. He had experienced thrombophlebitis at least 20 times during a 23-year period. He started to undergo hemodialysis in 1993, when he entered the end-stage of renal failure, probably due to Fabry's disease. He had multiple brain infarctions in the centrum semiovale and basal ganglia. Patient 2 had the typical manifestations of classic Fabry's disease, including severe pain and paresthesia in the distal extremities, angiokeratoma, proteinuria and mild renal dysfunction. He had multiple brain infarctions in the basal ganglia and pons. The diagnoses were confirmed by cranial CT and MRI. We observed similar multiple brain infarctions in Patients 1 and 3.

In this $\alpha$-Gal gene analysis of 36 Fabry's disease families, a variety of mutations causing Fabry's disease was identified: 6 partial deletions (8-10), 3 splicing mutations $(9,11,12), 13$ missense mutations $(2,12-15)$ and 4 nonsense mutations (15, 16). Two unrelated families had the same gene mutation (16). Direct sequencing analysis did not reveal any mutations in the $\alpha$-Gal coding region in the remaining 9 families. Northern blot analysis was performed on 5 of the families, which revealed that the amount of $\alpha$-Gal mRNA was markedly decreased in the patients (2). Some mutations might be outside the coding region involved in the transcription of $\alpha-\mathrm{Gal}$ in these patients.

\section{Discussion}

We demonstrated that the incidence of thrombosis is high in

Table 1. Profiles of Patients with Fabry's Disease who Developed Thrombosis

\begin{tabular}{|c|c|c|c|c|c|c|c|}
\hline 3 & 54 & $\mathrm{M}$ & Del 3b (\#1072-1074) & 0.1 & $\begin{array}{l}\text { Multiple brain infarctions } \\
\text { (White matter) }\end{array}$ & 50 & New case \\
\hline 4 & 59 & M & Q357X $(\mathrm{CAG} \rightarrow \mathrm{TAG})$ & 0.3 & $\begin{array}{l}\text { Recurrent thrombophlebitis, } \\
\text { Multiple brain infarctions } \\
\text { (Centrum semiovale, Basal ganglia) }\end{array}$ & $\begin{array}{c}35-59 \\
57\end{array}$ & New case \\
\hline $6^{\S}$ & 53 & $\mathrm{~F}$ & Del 13b (\#125-137) & 19.1 & Brain infarctions & 43 & 3 \\
\hline $7^{\S}$ & 60 & $\mathrm{~F}$ & Del 13b (\#125-137) & 10.5 & Brain infarctions & $49-59$ & 3 \\
\hline
\end{tabular}

*: Numbered according to Bishop et al (Ref. 19). ${ }^{\dagger}$ : Leukocyte $\alpha$-Gal activity is expressed as a percentage of the control mean level. ${ }^{\ddagger}$ : Distributions are shown in parentheses. ${ }^{\S}$ : Patients 6 and 7 are sisters. Patient 7 developed recurrent attacks of left-hemiparesis and died of cardiac failure at 60 years of age. 


\section{Thrombosis in Fabry's Disease}

patients with Fabry's disease. Grewal recently reported that strokes are a frequent complication of this disease (5). In the present study, various kinds of thrombotic accidents, such as strokes, central retinal artery occlusion and thrombophlebitis, were found to have occurred not only in hemizygous males but also in heterozygous females. This suggested that general blood vessels were affected by the disease process in both hemizygotes and heterozygotes. Pathological studies demonstrated the accumulation of globotriaosylceramide in the endothelial cells of blood vessels. These cells, often accompanied by endothelial cell proliferation, encroach on the lumen, causing a focal increase in intraluminal pressure, dilatation and angioectasis, which may lead to thrombosis of the brain and other tissues. There were no other potential etiological factors for the development of thrombosis, such as atrial fibrillation, mitral insufficiency, hypertension or hyperlipidemia, in the patients described here except for Patients 6 and 7 sisters who had mitral valve prolapse.

Although the leukocyte $\alpha$-Gal activity of variant form hemizygotes was lower than that of heterozygous females with thrombosis, none of the variant form hemizygotes experienced thrombotic accidents. At the cellular level, heterozygous females with classic Fabry's disease are predicted to have two populations of cells, one with deficient $\alpha$-Gal activity and the other with normal enzyme activity $(17,18)$, resulting from random X-chromosome inactivation (18). In contrast, hemizygous males with the variant Fabry's disease are believed to have residual $\alpha$-Gal activity in all cells. Some heterozygotes with the classic form of the disease exhibiting deficient $\alpha-G a l$ activity in many endothelial cells may be more affected by thrombosis than hemizygotes with the variant form.

The mutations in the thrombotic patients were variable and not restricted to any part of the $\alpha$-Gal gene, suggesting that the thrombotic tendency was unrelated to the type or location of the mutation.

We demonstrated a high incidence of $12 \%$ of thrombosis in Fabry's disease. The complication of thrombotic accidents should be taken into account in patients with Fabry's disease. This disease should also be considered as a cause of unexplained thrombotic accidents.

\section{References}

1) Desnick RJ, Ioannou YA, Eng CM. $\alpha$-galactosidase A deficiency: Fabry disease. in: The Metabolic and Molecular Basis of Inherited Disease, Scriver CR, Beaudet AL, Sly WS, Valle D, Eds. 7th ed., McGraw-Hill, New York, 1995, p. 2741.

2) Nakao S, Takenaka T, Maeda M, et al. An atypical variant of Fabry's disease in men with left ventricular hypertrophy. New Engl J Med 333:
$288,1995$.

3) Sakuraba H, Yanagawa Y, Igarashi T, et al. Cardiovascular manifestations in Fabry's disease. A high incidence of mitral valve prolapse in hemizygotes and heterozygotes. Clin Genet 29: 276, 1986.

4) Fukushima M, Tsuchiyama $Y$, Nakato $T$, et al. A female heterozygous patient of Fabry's disease with renal accumulation of trihexosylceramide detected with a monoclonal antibody. Am J Kidney Dis 26: 952, 1995.

5) Grewal RP. Stroke in Fabry's disease. J Neurol 241: 153, 1994.

6) Mayes JS, Scheerer JB, Sifers RN, Donaldson ML. Differential assay for lysosomal $\alpha$-galactosidases in human tissues and its application to Fabry's disease. Clin Chim Acta 112: 247, 1981.

7) Ishii $S$, Kase R, Sakuraba H, et al. Human $\alpha$-galactosidase gene expression: significance of two peptide regions encoded by exons 1-2 and 6 . Biochim Biophys Acta 1204: 265, 1994.

8) Fukuhara Y, Sakuraba H, Oshima A, et al. Partial deletion of human $\alpha$ galactosidase A gene in Fabry disease: direct repeat sequences as a possible cause of slipped mispairing. Biochem Biophys Res Commun 170: 296, 1990.

9) Okumiya T, Takenaka T, Ishii S, Kase R, Kamei S, Sakuraba H. Two novel mutations in the $\alpha$-galactosidase gene in Japanese classical hemizygotes with Fabry disease. Jpn J Hum Genet 41: 313, 1996.

10) Takenaka $T$, Sakuraba H, Hashimoto $K$, et al. Coexistence of gene mutations causing Fabry disease and Duchenne muscular dystrophy in a Japanese boy. Clin Genet 49: 255, 1996.

11) Sakuraba H, Eng CM, Desnick RJ, Bishop DF. Invariant exon skipping in the human $\alpha$-galactosidase A pre-mRNA: $\mathrm{A} \mathrm{g}^{+1}$ to $t$ substitution in a 5 'splice site causing Fabry disease. Genomics 12: 643, 1992.

12) Okumiya $T$, Ishii $S$, Kase R, Kamei $S$, Sakuraba H, Suzuki Y. $\alpha$ Galactosidase gene mutations in Fabry disease: heterogeneous expressions of mutant enzyme proteins. Hum Genet 95: 557, 1995.

13) Ishii $S$, Sakuraba H, Suzuki Y. Point mutations in the upstream region of the $\alpha$-galactosidase A gene exon 6 in an atypical variant of Fabry disease. Hum Genet 89: 29, 1992.

14) Koide $T$, Ishiura $M$, Iwai $K$, et al. A case of Fabry's disease in a patient with no $\alpha$-galactosidase A activity caused by a single amino-acid substitution of Pro-40 by Ser. FEBS Lett 259: 353, 1990.

15) Takata $T$, Okumiya $T$, Hayashibe $H$, et al. Screening and detection of gene mutations in Japanese patients with Fabry disease by non-radioactive single-stranded conformation polymorphism analysis. Brain Dev 19: 111, 1997.

16) Sakuraba H, Oshima A, Fukuhara $Y$, et al. Identification of point mutations in the $\alpha$-galactosidase A gene in classical and atypical hemizygotes with Fabry disease. Am J Hum Genet 47: 784, 1990.

17) Itoh $K$, Kotani M, Tai $T$, et al. Immunofluorescence imaging diagnosis of Fabry heterozygotes using confocal laser scanning microscopy. Clin Genet 44: 302, 1993.

18) Lyon MF. Sex chromatin and gene action in the mammalian X-chromosome. Am J Hum Genet 14: 135, 1962.

19) Bishop DF, Kornreich R, Eng CE, Ioannou YA, Fitzmaurice TF, Desnick RJ. Human $\alpha$-galactosidase: Characterization and eukaryotic expression of the full-length cDNA and structural organization of the gene. in: Lipid Storage Disorders: Biological and Medical Aspects, Salvayre R, DousteBlazy L, Gatt S, Eds. New York, Plenum, 1988, 809.

20) Uyama E, Ueno N, Uchino M, et al. Headache associated with aseptic meningeal reaction as clinical onset of Fabry's disease. Headache 35: 498, 1995. 